\title{
A Multi-criteria Decision Making Method Based on Linguistic Preference Infor- mation for IT Outsourcing Vendor Selec- tion in Hospitals
}

\author{
Wenhui Liu ${ }^{1}$ Quan $\mathrm{Li}^{2}$ \\ ${ }^{1}$ Hospital of North China Electric Power University, Baoding, P.R.China \\ ${ }^{2}$ Department of Economics and Management, North China Electric Power University \\ Baoding, P.R.China
}

\begin{abstract}
The decision of IT outsourcing in hospitals requires close attention to the evaluation of vendor selection process because the selection decision involves conflicting multiple criteria and is replete with complex decision-making problems. Based on Extended Ordered Weighted Averaging (EOWA) operator, a method of multicriteria decision making with linguistic preference information is introduced to the problem. An illustrative numerical example is also provided to illustrate the feasibility and practicality of the proposed method.
\end{abstract}

Keywords: IT outsourcing vendor; multicreteria decision making; linguistic preference information; Extended Ordered Weighted Averaging operator

\section{Introduction}

Problems like increasing costs, insufficient capacity due to aging of population and inefficient ways of working will lead to new ways of thinking within hospitals [1]. The successful adoption of Information Technology could provide a hospital with a vessel to decrease spending and improve quality. The impact of IT could be seen from many processes with- in hospitals. However, managing, implementing and developing IT do not belong to the core competences of a hospital. One solution regarding the adoption of IT could be the decision to outsource[2]. The decision-making process around IT outsourcing in a hospital is difficult and comprehensive. A lot of factors should be taken into thorough consideration in the process. It requires close attention to the evaluation of vendor selection process because the selection decision involves conflicting multiple criteria and is replete with complex decision-making problems[3]. Selecting the most appropriate vendor is considered an important strategic decision that may impact the performance of outsourcing engagements. While with the uncertainty of internal and external environment and the complexity of the relationships with outsourcing vendors, there might be many risks when a hospital wants to choose an IT outsourcing vendor that best fits its need. Hence, it is quite necessary for a hospital to utilize some decision making tool to fufill that purpose. Meanwhile, the indices used for the evalution of IT outsourcing vendors are mostly qualitative, a hospital often has to face with the problems of fuzziness of the indices. To deal with such problems, this paper introduces a multicriteria decision making(MCDM) method 
based on linguistic preference information to solving such problems. This paper is structured as follows. In Section 2 , the multi-criteria decision making method and an operator EOWA are introduced. In Section 3, a method, based on MCDM and EOWA operator, is introduced to ranking IT outsourcing vendors. In Section 4, an numerical example is provided to illustrate the proposed method. Finally, in Section 5, some conclusions are drawn.

\section{Theoretical Background}

\subsection{Multi-criteria Decision Making.}

In a real-world decision process, a decision maker is often faced with complex decision environments and problems. Sometimes there are many factors of the problems, and the interrelationships among the factors are very complicated. Relationships between factors of a problem may be highly nonlinear; changes in the elements may not be related by simple proportionality. This is essentially a problem of Multi-criteria decision making (MCDM), which deal mainly with problems about evaluation or selection. MCDM approaches seek to take explicit account of more than one criterion in supporting the decision process. A complete MCDM process should involve the following elements: criterion set, preference structure, alternative set, and performance values. MCDM is useful in circumstances which necessitate the consideration of different courses of action, which can not be evaluated by the measurement of a simple, single dimension. Due to the above reason, MCDM is naturally utilized to the evaluation process of IT outsourcing vendors.

MCDM process is characterized by a decision maker, who is asked to provide his or her own preferences on a predefined set of alternatives with respect to each criterion in a predefined set of crite- ria. In some cases, due to reasons like time pressure, lack of knowledge or data, or limited expertise related with problem domain [4], the decision maker might only provide the information with a linguistic term. Further, in some situations, the decision maker tends to provide the information with linguistic preferences. $\mathrm{Xu}$ has applied an extended ordered weighted averaging (EOWA) operator to multicriteria decision making with linguistic information [6].

\subsection{Representation of MCDM with Uncertain Linguistic Information.}

Let $S=\left\{s_{i} \mid i=-t, \ldots, t\right\}$ be a finite and totally ordered discrete label set. Any label, $S_{i}$, represents a possible value for a linguistic variable, and among the others, it has the following two characteristics [5]:

- The set is ordered: $s_{i}>s_{j}$ if $i>j$;

- There is the negation operator: $\operatorname{neg}\left(s_{i}\right)=s_{-i}$.

In a way, $\mathrm{S}$ can be defined as follows:

$$
\begin{aligned}
S=\left\{s_{-4}\right. & =\text { extremely poor, } s_{-3}=\text { very poor, } \\
s_{-2} & =\text { poor }, s_{-1}=\text { slightly poor, } s_{0}=\text { fair }, \\
s_{1} & =\text { slightly good, } s_{2}=\text { good }, \\
s_{3} & \left.=\text { very good, } s_{4}=\text { extremely good }\right\}
\end{aligned}
$$

preserve all the given information, $\mathrm{Xu}$ has extended $S$ to a continuous label set $\bar{S}=\left\{s_{a} \mid a \in[-q, q]\right\}$, where $q(q>t)$ is a sufficiently large positive integer. If $s_{\alpha} \in S$, then $s_{\alpha}$ is called original label, otherwise, it's called virtual label. In general, the decision makers would utilize the original linguistic labels to evaluate alternatives, and the virtual linguistic labels might only appear in calculation. 
For any two labels $s_{\alpha}, s_{\beta} \in \bar{S}$, we have two operational laws:

$$
s_{\alpha} \oplus s_{\beta}=s_{\alpha+\beta} ;
$$$$
\mu s_{\alpha}=s_{\mu \alpha}, \text { where } \mu \in[0,1] \text {. }
$$

\subsection{Extended Ordered Weighted Av- eraging (EOWA) Operator}

An EOWA operator of dimension $n$ is a mapping: $\bar{S}^{n} \rightarrow \bar{S}$ that has associated a weighting vector $w=\left(w_{1}, w_{2}, \ldots, w_{n}\right)$ to it, such that $w_{j} \in[0,1], \sum_{j=1}^{n} w_{j}=1$, and is defined to aggregate a collection of linguistic variables $s_{\alpha_{1}}, s_{\alpha_{2}}, \ldots, s_{\alpha_{n}}$ according to the following expression: $\operatorname{EOWA}_{w}\left(s_{\alpha_{1}}, s_{\alpha_{2}} \ldots, s_{\alpha_{n}}\right)$ $=w_{1} s_{\beta_{1}} \oplus w_{2} s_{\beta_{2}} \oplus \ldots \oplus w_{n} s_{\beta_{n}}$ $=s_{\bar{\beta}}$

where $\bar{\beta}=\sum_{j=1}^{n} w_{j} \beta_{j}, s_{\beta_{j}}$ is the $j^{\text {th }}$ largest of the $S_{\alpha_{i}}$.

\section{A Method for Evaluation of IT Out- sourcing Vendors Based on MCDM with Linguistic Preference Infor- mation}

In this section, a method for evaluation of IT outsourcing vendors based on MCDM with linguistic preference information is proposed as follows:

Step 1. For an evaluation of IT outsourcing vendors based on MCDM with linguistic preference information, let $X=\left\{x_{1}, x_{2}, \ldots, x_{n}\right\}$ be the set of alternatives, $C=\left\{c_{1}, c_{2}, \ldots, c_{v}\right\}$ be the set of criteria. First, the decision maker compares these alternatives with respect to each criterion by the linguistic terms in the set $S=\left\{s_{i} \mid i=-t, \ldots, t\right\}$, and then constructs the linguistic preference relation matrix $R=\left(r_{i j}\right)_{n \times m}$, where $r_{i j} \in S$.

Step 2. Utilize the EOWA operator $z_{i}(w)=E O W A_{w}\left(r_{i 1}, r_{i 2}, \cdots, r_{i m}\right)$

$i, j=1,2, \ldots, n \quad$ to aggregate $r_{i j}(j=1,2, \ldots, n)$ corresponding to the alternative $x_{i}$ with respect to each criterion, and then get all the averaged preference degrees $z_{i}(\omega)(i \in N)$ of all the other alternatives.

Step 3. Rank all the alternatives and select the best one(s) in accordance with the values of $z_{i}(\omega)(i \in N)$. The alternative which has the biggest value of $z_{i}(\omega)(i \in N)$ is the best one.

\section{An Numerical Example}

The purpose of vendor selection is to determine the optimal IT service vendor who will offer the best all-around package of products and services, but selecting the right vendor is influenced by a variety of factors. In this paper, ten key criteria were selected as follows: $u_{1}$ : Technical capability; $u_{2}$ : Financial performance; $u_{3}$ : Performance history; $u_{4}$ : Quality; $u_{5}$ : Price; $u_{6}$ : Flexibility $; u_{7}$ : Reputation; $u_{8}$ : Delivery time; $u_{9}$ : Experience; $u_{10}$ : Market share.

Let's suppose that there are four IT outsouncing vendors $x_{i}=(i=1,2,3,4)$ available to be ranked. Here, we assume that the weighting vector of the criteria is $\omega=(0.07,0.08,0.10,0.12,0.13,0.13,0.12$, $0.10,0.08,0.07)$. The linguistic labels used are shown in the following set: $S=\left\{s_{-5}, \cdots, s_{5}\right\}=\{$ extremely poor, very poor, poor, a little poor, slightly poor, fair, 
slightly good, a little good, good, very good, extremely good \}.

Step 1. The decision maker compares the four alternatives with respect to the ten criteria and constructs the linguistic preference relations as shown in Table 1.

Table 1. Linguistic preference relation

\begin{tabular}{c|cccccccccc}
\hline & $u_{1}$ & $u_{2}$ & $u_{3}$ & $u_{4}$ & $u_{5}$ & $u_{6}$ & $u_{7}$ & $u_{8}$ & $u_{9}$ & $u_{10}$ \\
\hline$x_{1}$ & $s_{2}$ & $s_{2}$ & $s_{0}$ & $s_{0}$ & $s_{0}$ & $s_{3}$ & $s_{3}$ & $s_{4}$ & $s_{2}$ & $s_{-1}$ \\
$x_{2}$ & $s_{3}$ & $s_{0}$ & $s_{-2}$ & $s_{3}$ & $s_{3}$ & $s_{4}$ & $s_{3}$ & $s_{2}$ & $s_{-1}$ & $s_{2}$ \\
$x_{3}$ & $s_{2}$ & $s_{3}$ & $s_{4}$ & $s_{4}$ & $s_{2}$ & $s_{2}$ & $s_{2}$ & $s_{3}$ & $s_{2}$ & $s_{0}$ \\
$x_{4}$ & $s_{4}$ & $s_{3}$ & $s_{3}$ & $s_{0}$ & $s_{3}$ & $s_{0}$ & $s_{3}$ & $s_{3}$ & $s_{0}$ & $s_{-1}$ \\
\hline
\end{tabular}

Step 2. Utilize the EOWA operator $r_{i}=E O W A_{w}\left(r_{i 1}, r_{i 2}, r_{i 3}, r_{i 4}, r_{i 5}, r_{i 6}, r_{i 7}, r_{i 8}, r_{i 9}, r_{i 10}\right)$ to aggregate $r_{i j}(j=1,2,3,4,5,6,7,8,9,10)$

corresponding to the alternative $x_{i}$ with respect to each criterion, and then get all the averaged preference degrees of all the alternatives. The result is as follows:

$$
\begin{aligned}
& z_{1}(\omega)=0.07 \times s_{4} \oplus 0.08 \times s_{3} \oplus 0.10 \times s_{3} \\
& \oplus 0.12 \times s_{2} \oplus 0.13 \times s_{2} \oplus 0.13 \times s_{2} \\
& \oplus 0.12 \times s_{0} \oplus 0.10 \times s_{0} \oplus 0.08 \times s_{0} \\
& \oplus 0.07 \times s_{-1}=s_{1.51} \\
& z_{2}(\omega)=0.07 \times s_{4} \oplus 0.08 \times s_{3} \oplus 0.10 \times s_{3} \\
& \oplus 0.12 \times s_{3} \oplus 0.13 \times s_{3} \oplus 0.13 \times s_{2} \\
& \oplus 0.12 \times s_{2} \oplus 0.10 \times s_{0} \oplus 0.08 \times s_{-1} \\
& \oplus 0.07 \times s_{-2}=s_{1.85} \\
& z_{3}(\omega)=0.07 \times s_{4} \oplus 0.08 \times s_{4} \oplus 0.10 \times s_{3} \\
& \oplus 0.12 \times s_{3} \oplus 0.13 \times s_{2} \oplus 0.13 \times s_{2} \\
& \oplus 0.12 \times s_{2} \oplus 0.10 \times s_{2} \oplus 0.08 \times s_{2} \\
& \oplus 0.07 \times s_{0}=s_{2.38} \\
& z_{4}(\omega)=0.07 \times s_{4} \oplus 0.08 \times s_{3} \oplus 0.10 \times s_{3} \\
& \oplus 0.12 \times s_{3} \oplus 0.13 \times s_{3} \oplus 0.13 \times s_{3} \\
& \oplus 0.12 \times s_{0} \oplus 0.10 \times s_{0} \oplus 0.08 \times s_{0} \\
& \oplus 0.07 \times s_{-1}=s_{1.89}
\end{aligned}
$$

Step 3. Rank all the alternatives and select the best one(s) in accordance with the values of $z_{i}(\omega)(i=1,2,3,4)$ :

$z_{3}(\omega) \succ z_{4}(\omega) \succ z_{2}(\omega) \succ z_{1}(\omega)$, so the best alternative is $x_{3}$.

\section{Summary}

In this paper, the problem of evalution of IT outsourcing vendors is studied. Based on EOWA operator and multi-criteria decision making with linguistic preference information, a method is introduced to the problem. An illustrative numerical example is also provided to illustrate the feasibility and practicality of the proposed method. The proposed method is straightforward and can effectively avoid the loss of information.

\section{References}

[1] CGI Whitepaper (2002), "IT outsourcing for healthcare: It's time to seriously consider," CGI Group.

[2] Lorence, D.P. \& Spink, A., " Healthcare information systems outsourcing, " International Journal of Information Management, Vol24, No.2, pp. 131-145, 2004

[3] Yuang, C. \& Huang, J.B., "A decision model for IS outsourcing," International Journal of Information Management, Vol20, No.3, pp. 225239, 2000.

[4] M. Weber, "Decision making with incomplete information," European Journal of Operational Research, Vol28, No.1 , pp. 44-57, 1987.

[5] Z. S. Xu, "Deviation measures of linguistic preference relations in group decision making," Omega, Vol 33, No.3, pp. 249-254, 2005.

[6] Z. S. Xu, Uncertain multiple attribute decision making: methods and applications, Tsinghua University Press, Beijing (2004). 